\title{
Pharmacogenomics of anticoagulation therapy: the last 10 years
}

\author{
Georgia Ragia1,2 \& Vangelis G Manolopoulos*,1,3 \\ ${ }^{1}$ Laboratory of Pharmacology, Medical School, Democritus University of Thrace, Alexandroupolis, Greece \\ ${ }^{2}$ DNALEX SA, Leontaridou 2, Alexandroupolis, Greece \\ ${ }^{3}$ Clinical Pharmacology \& Pharmacogenetics Unit, Academic General Hospital of Alexandroupolis, Alexandroupolis, Greece \\ *Author for correspondence: Tel./Fax: +30 255103 0523; emanolop@med.duth.gr
}

\begin{abstract}
"What once was a nightmare for treating clinicians (dosing patients with coumarins, due to fear of severe and often life-threatening bleeding) may soon become a gratifying routine, with the availability of both, several therapeutic choices (already available), and reliable genetic and epigenetic markers to guide therapy selection with the necessary precision."
\end{abstract}

First draft submitted: 11 October 2019; Accepted for publication: 14 October 2019; Published online: 22 November 2019

Keywords: acenocoumarol • algorithm • apixaban $\bullet$ coumarinic oral anticoagulants $\bullet$ dabigatran $\bullet$ edoxaban $\bullet$ epigenetics $\bullet$ epigenomics $\bullet$ non-VKA oral anticoagulants $\bullet$ pharmacoepigenomics $\bullet$ pharmacogenomics $\bullet$ rivaroxaban - warfarin

10 years ago, we were discussing in this journal the past, present and anticipated future of coumarinic oral anticoagulant (COAs) pharmacogenomics [1]. Already then, evidence was strong in support of the use of pharmacogenetic information on specific CYP2C9 and VKORC1 polymorphisms to guide anticoagulation with coumarinic drugs. However, this evidence derived from retrospective studies, and the field suffered from the lack of randomized prospective clinical trials (RCTs).

Since 2009, six main developments have occurred in this field: several RCTs aiming to assess the safety and clinical utility of newly developed pharmacogenomic-guided dosing algorithms for warfarin, acenocoumarol and phenprocoumon were completed and published; data accumulated on the cost-effectiveness of pharmacogenomic-guided dosing strategy; the extended use of algorithms led to the realization that ethnic specific algorithms are necessary; CYP4F2 was established as an additional (albeit minor) marker of response; the new anticoagulants dabigatran, rivaroxaban, apixaban and most recently edoxaban have gradually dominated over COAs; and understanding of the role of epigenetics in anticoagulation has gained increased attention and raised expectations for the generation of novel markers to help improve precision in anticoagulation pharmacotherapy. Below, we briefly discuss each of these developments and cap this editorial with some conclusions and future perspectives.

\section{The completion \& publication of randomized clinical trials}

November 2013 can be considered a hallmark of COA RCTs: the results of three large RCTs (Clarification of Optimal Anticoagulant through Genetics [COAG] and The European Pharmacogenetics of Anticoagulant Therapy [EU-PACT]) for all three COAs were published in New England Journal of Medicine [2-4]. These RCTs began in 2010 with the aim of assessing the safety, clinical utility and cost-effectiveness of newly developed CYP2C9/VKORC1 pharmacogenomic-guided dosing algorithms for COAs. In the COAG trial, genotype-guided dosing of warfarin did not improve anticoagulation control during the first 4 weeks of therapy [2]. The EU-PACT warfarin trial results showed that pharmacogenomic-based dosing was associated with a higher percentage of time in the therapeutic international normalized ratio (INR) range (PTIR) than was standard dosing during the initiation of warfarin therapy [3]. EU-PACT acenocoumarol and phenprocoumon trial results showed a significant improvement in PTIR in the first 4 weeks in the pharmacogenomic dosing arm; however, no statistically significant difference in the primary outcome, the PTIR in the first 12 weeks, was observed between trial arms [4].

For acenocoumarol and phenprocoumon, apart from the EU-PACT trial other RCTs are missing. For warfarin, several other prospective trials were published (ClinicalTrials.gov Identifier NCT01178034, NCT02065388,

Future Medicine 
NCT01006733, NCT00700895 in [5]) and almost all have shown positive results. The largest, so far, published RCT is the 'Genetic Informatics Trial of Warfarin to Prevent Deep Vein Thrombosis' trial with a total of 1597 patients (808 randomized in the pharmacogenomic arm and 789 in the clinically guided arm) treated with warfarin to prevent deep vein thrombosis after hip or knee arthroplasty [6]. Results showed that the rate of a composite of major bleeding, INR of 4 or greater, venous thromboembolism (VTE), or death in patients undergoing hip or knee arthroplasty was reduced in genotype-guided warfarin dosing arm that included CYP2C9, VKORC1, and CYP4F2 genotype information, compared with clinically guided warfarin dosing arm [6].

\section{Cost-effectiveness of pharmacogenomic-guided dosing strategy}

At least 16 studies have been published assessing the cost-effectiveness of pharmacogenomic-guided dosing for warfarin and, overall, results provide supportive evidence that this strategy is cost-effective [7]. However, results are diverse with less support for cost-effectiveness from the societal and provider perspectives. Data are also available from EU-PACT cost-effectiveness analyses [8,9]. Pharmacogenomic-guided dosing of warfarin was a costeffective strategy to improve outcomes of patients with atrial fibrillation treated with warfarin in the UK and in Sweden [8]. Cost-effectiveness Dutch EU-PACT data analysis for acenocoumarol and phenprocoumon has shown that pharmacogenomic dosing improves health only slightly when compared with clinical dosing [9]. It should be kept in mind, however, that the cost of genotyping is constantly reducing and in the near future relevant genotype information may be readily available for millions of patients in many countries without additional cost. This would change economic aspects of anticoagulant pharmacogenomics dramatically and make it a clearly cost-effective option.

\section{Ethnic specific algorithms are necessary}

Accumulated evidence indicates that a universal dosing algorithm of COAs may not have optimal performance for different populations. These ethnicity-based differences could be explained considering that the frequency of the VKORC1 and CYP2C9 alleles varies between different ethnic groups; therefore, rare variants should be taken under consideration for different populations. Such a variant is in CYP2C rs12777823 locus present in African Americans [10]. Indeed, the COAG trial included 27\% African-Americans [2] and raised the issue of rare variant genotyping in specific populations. Similarly, when analyzing the EU-PACT acenocoumarol trial data, we have shown that the dosing algorithm used (developed in The Netherlands in a Dutch population) does not fit as well as anticipated to the Greek population [11]. Data available for warfarin for which an international pharmacogenetic algorithm is available online and can be freely used [12] also show that the accuracy of the international pharmacogenetic algorithm is not sufficient to be used for warfarin screening in Turkish, Brazilian or Han Chinese patients [13-15].

\section{The establishment of CYP4F2 as an additional marker of response}

Despite the intense research on the missing heritability of COA pharmacogenomics, hitherto only the CYP4F2 gene was found in genome-wide association studies (GWAS) to contribute, to a much lesser extent (approximately 1-2\%) to $\mathrm{COA}$ dose prediction $[16,17]$. CYP4F2*3 allele decreases CYP4F2 activity, preventing inactivation of vitamin $\mathrm{K}$ and thus explaining the need for higher COA dosages $[16,17]$. For Caucasians, there is lack of evidence in GWAS scale for other genes that could be associated with COA variable response or dose requirements, suggesting that additional major genetic predictors may not exist or may not emerge in the near-term. Nevertheless, CYP4F2 association should be further assessed as for its effect on the intervariable response observed within distinct CYP2C9/VKORC1 genotypic groups. Toward this aim, we have previously shown that significant CYP4F2 effects were found on patient ability to reach stable dose and on acenocoumarol dose requirements when CYP2C9/VKORC1 subphenotypes were analyzed [18].

\section{Gradual dominance of the new anticoagulants}

The last decade has seen a major shift in oral anticoagulation; therapeutic options for nonvalvular atrial fibrillation, deep vein thrombosis and other indications now include several other drugs collectively known as non-VKA oral anticoagulants (NOACs) which directly inhibit coagulation factors. Dabigatran etexilate, a direct thrombin inhibitor, was the first NOAC to gain market approval in 2010, followed by three direct factor Xa inhibitors, namely rivaroxaban (2011), apixaban (2012) and edoxaban (2015). 
The prescription of NOACs has increased dramatically since their introduction. Apixaban and rivaroxaban were among the ten best selling drugs for 2018 also seeing sales increases from 2017. More specifically, apixaban is the second highest selling drug (33.5\% sales increase) and rivaroxaban is number 10 (5.8\% increase). 2018 sales of these two NOACs exceeded 16 billion dollars [19]. Interestingly, the increase in NOAC prescription rates has so far not been accompanied by a similar increase in known pharmacogenomic traits that affect NOAC response.

Notably, except for a GWAS performed in patients treated with dabigatran [20], there are no other genome-wide pharmacogenomic studies on NOACs. Currently, only a few genes have been studied in association with NOAC response in humans (patient cohorts or healthy volunteers) [21]: $C E S 1$ and $A B C B 1$ for dabigatran, $A B C B 1$ and $C Y P 3 A 4 / 5$ for rivaroxaban, $A B C B 1, A B C G 2$ and $C Y P 3 A 4 / 5$ for apixaban, and factor $X, A B C B 1$ and SLCO1B1 for edoxaban. NOAC pharmacogenomics is still a naive albeit emerging field of research; thus, firm conclusions have not yet been drawn and all derived results need to be replicated in different populations.

In anticoagulation therapy, currently, COA pharmacogenomics has a twofold dimension, both predicting the optimal dosing of COAs and identifying individuals who are at increased risk of bleeding with these drugs and, consequently, would benefit from a NOAC rather than COAs [22,23]. However, further developments for anticoagulation pharmacogenomics are expected for both COAs and NOACs.

\section{Epigenetics in oral anticoagulation}

Epigenetic modifications refer to post-translational modifications of histone proteins, DNA methylation, chromatin remodeling and noncoding RNAs, such as miRNAs. Regarding COAs, some epigenetic findings have been reported, and they are limited to warfarin. In a genome-wide methylation study, Luo et al. identified eight probes with significant methylation change associated with extreme warfarin dosage in individuals with VKORC1 -1639AA and $C Y P 2 C 9^{*} 1 /^{*} 1$ genotypes [24]. However, they did not identify a CpG site that was significantly associated with warfarin dosage after correction or using of p-value threshold. Regarding miRNAs, at first it was shown that miR-133 regulates VKORC1 expression and this regulation may influence anticoagulant therapy with COAs [25]. Subsequently, it was reported that polymorphisms within miR-133 encoding genes hold the potential to affect warfarin dosing [26]. Recently, Kabiri Rad et al. showed that the rate of miR-133a-3p expression was higher in patients with heart valve replacement receiving warfarin compared with controls [27].

Future targets for oral anticoagulant pharmaco(epi)genomic analyses can be hypothesis driven. For COAs, solid epigenetic-based hypotheses can be made from published data. In silico analysis has provided information on the potential association of GATA-4 transcription factor with CYP2C9 expression [28]. This GATA-mediated regulation of CYP2C9 might be, to some extent, responsible for the interindividual variability in CYP2C9 enzymatic activity at individuals who carry the wild-type genotype of CYP2C9. Additionally, mediator subunit MED25 has been proposed as a central regulatory factor of CYP2C9 expression through histone acetylation and chromatin remodeling pathways [29]. miRNAs that are associated with CYP2C9 regulation are also attractive candidates for COA pharmacoepigenomic analyses: miR130b downregulates CYP2C9 enzyme activity by at least 30\% [30], whereas hsa-miR128-3p suppresses CYP2C9 expression in human hepatocellular carcinoma cells [31]. These results may be extended to COA efficacy and dosing variability.

For NOACs, genes involved in pharmacokinetics and pharmacodynamics of the drugs appear as attractive candidates. Additionally, epigenetic modifications associated with response to ablation-based atrial fibrillation treatment [32-37] could also be associated with NOAC response. Similarly, for VTE, miRNAs involved in VTE pathogenesis or associated with the risk of VTE recurrence could potentially interfere with NOAC response [38,39].

\section{Conclusion \& future perspective}

In summary, it appears that a significant body of the necessary evidence is available for COA pharmacogenomics to be implemented into clinical routine. In reality, the extent to which the pharmacogenomic-guided strategy in coumarin anticoagulation is currently adopted by hospitals around the world is unknown and appears to be limited. We anticipate that the next decade will bring several developments in oral anticoagulant pharmacogenomics, for both COAs and NOACs. With the availability of genotype information for most patients, we expect that using this information to guide anticoagulation pharmacotherapy will be the norm. This will help COAs remain in the therapeutic arsenal for anticoagulation as a rational choice among available anticoagulants to increase drug safety and reduce health system costs. Finally, for both COAs and NOACs, we suggest that the pharmaco(epi)genomics field, still at its infancy in anticoagulation, holds the potential not only to identify novel (epi)genetic markers associated with response to these drugs, but also to shed light into distinct pathways and specific molecules that 
could serve as easily accessible biomarkers for NOAC response monitoring. We believe that the future of precision pharmacotherapy for both old and new anticoagulant drugs is bright and will bring several significant developments. What once was a nightmare for treating clinicians (dosing patients with coumarins, due to fear of severe and often life-threatening bleeding) may soon become a gratifying routine, with the availability of both, several therapeutic choices (already available), and reliable genetic and epigenetic markers to guide therapy selection with the necessary precision.

\section{Financial \& competing interests disclosure}

The authors have no relevant affiliations or financial involvement with any organization or entity with a financial interest in or financial conflict with the subject matter or materials discussed in the manuscript. This includes employment, consultancies, honoraria, stock ownership or options, expert testimony, grants or patents received or pending, or royalties.

No writing assistance was utilized in the production of this manuscript.

\section{References}

1. Manolopoulos VG, Ragia G, Tavridou A. Pharmacogenetics of coumarinic oral anticoagulants. Pharmacogenomics 11(4), 493-496 (2010).

2. Kimmel SE, French B, Kasner SE et al. A pharmacogenetic versus a clinical algorithm for warfarin dosing. N. Engl. J. Med. 369(24), 2283-2293 (2013).

3. Pirmohamed M, Burnside G, Eriksson N et al. A randomized trial of genotype-guided dosing of warfarin. N. Engl. J. Med. 369(24), 2294-2303 (2013).

4. Verhoef TI, Ragia G, de Boer A et al. A randomized trial of genotype-guided dosing of acenocoumarol and phenprocoumon. N. Engl. J. Med. 369(24), 2304-2312 (2013).

5. ClinicalTrials.gov. https://clinicaltrials.gov/ct2/home

6. Gage BF, Bass AR, Lin H et al. Effect of genotype-guided warfarin dosing on clinical events and anticoagulation control among patients undergoing hip or knee arthroplasty: the GIFT randomized clinical trial. JAMA 318(12), 1115-1124 (2017).

7. Zhu Y, Swanson KM, Rojas RL et al. Systematic review of the evidence on the cost-effectiveness of pharmacogenomics-guided treatment for cardiovascular diseases. Genet Med. doi: 10.1038/s41436-019-0667-y (2019) (Epub ahead of print).

8. Verhoef TI, Redekop WK, Langenskiold S et al. Cost-effectiveness of pharmacogenetic-guided dosing of warfarin in the United Kingdom and Sweden. Pharmacogenomics J. 16(5), 478-484 (2016).

9. Verhoef TI, Redekop WK, de Boer A, Maitland-van der Zee AH, EU-PACT group. Economic evaluation of a pharmacogenetic dosing algorithm for coumarin anticoagulants in The Netherlands. Pharmacogenomics 16(2), 101-114 (2015).

10. Perera MA, Cavallari LH, Limdi NA et al. Genetic variants associated with warfarin dose in African-American individuals: a genome-wide association study. Lancet 382(9894), 790-796 (2013).

11. Ragia G, Kolovou V, Kolovou G et al. A novel acenocoumarol pharmacogenomic dosing algorithm for the Greek population of EU-PACT trial. Pharmacogenomics 18(1), 23-34 (2017).

12. Warfarin Dosing. www. WarfarinDosing.org

13. Karaca S, Bozkurt NC, Cesuroglu T et al. International warfarin genotype-guided dosing algorithms in the Turkish population and their preventive effects on major and life-threatening hemorrhagic events. Pharmacogenomics 16(10), 1109-1118 (2015).

14. Santos PC, Marcatto LR, Duarte NE et al. Development of a pharmacogenetic-based warfarin dosing algorithm and its performance in Brazilian patients: highlighting the importance of population-specific calibration. Pharmacogenomics 16(8), 865-876 (2015).

15. Peng Q, Huang S, Chen X et al. Validation of warfarin pharmacogenetic algorithms in 586 Han Chinese patients. Pharmacogenomics 16(13), 1465-1474 (2015).

16. Takeuchi F, McGinnis R, Bourgeois $S$ et al. A genome-wide association study confirms VKORC1, CYP2C9, and CYP4F2 as principal genetic determinants of warfarin dose. PLoS Genet. 5(3), e1000433 (2009).

17. Teichert M, Eijgelsheim M, Rivadeneira F et al. A genome-wide association study of acenocoumarol maintenance dosage. Hum. Mol. Genet. 1, 3758-3768 (2009).

18. Ragia G, Karantza IM, Kelli-Kota E et al. Role of CYP4F2, CYP2C19, and CYP1A2 polymorphisms on acenocoumarol pharmacogenomic algorithm accuracy improvement in the Greek population: need for sub-phenotype analysis. Drug Metab. Pers. Ther. 32(4), 183-190 (2017).

19. Philippidis A. Top 15 best-selling drugs of 2018. Genet. Eng. Biotechnol. News 39(4) doi:10.1089/gen.39.04.07 (2019).

20. Paré G, Eriksson N, Lehr T et al. Genetic determinants of dabigatran plasma levels and their relation to bleeding. Circulation 127(13), $1404-1412$ (2013). 
21. Kanuri SH, Kreutz RP. Pharmacogenomics of novel direct oral anticoagulants: newly identified genes and genetic variants. J. Pers. Med. 9(1), pii: E7 (2019).

22. Mega JL, Walker JR, Ruff CT et al. Genetics and the clinical response to warfarin and edoxaban: findings from the randomised, double-blind ENGAGE AF-TIMI 48 trial. Lancet 385(9984), 2280-2287 (2015).

23. Vandell AG, Walker J, Brown KS et al. Genetics and clinical response to warfarin and edoxaban in patients with venous thromboembolism. Heart 103(22), 1800-1805 (2017).

24. Luo Z, Liu R, Sun B et al. Identification of gene modules associated with warfarin dosage by a genome-wide DNA methylation study. Pharmazie 73(5), 288-293 (2018).

25. Pérez-Andreu V, Teruel R, Corral J et al. miR-133a regulates vitamin K 2,3-epoxide reductase complex subunit 1 (VKORC1), a key protein in the vitamin K cycle. Mol. Med. 18, 1466-1472 (2013).

26. Ciccacci C, Rufini S, Politi C, Novelli G, Forte V, Borgiani P. Could microRNA polymorphisms influence warfarin dosing? A pharmacogenetics study on mir133 genes. Thromb. Res. 136(2), 367-370 (2015).

27. Kabiri Rad H, Mazaheri M, Dehghani Firozabadi A. Relative expression of PBMC microRNA-133a analysis in patients receiving warfarin after mechanical heart valve replacement. Avicenna J. Med. Biotechnol. 10(1), 29-33 (2018).

28. Mwinyi J, Nekvindová J, Cavaco I et al. New insights into the regulation of CYP2C9 gene expression: the role of the transcription factor GATA-4. Drug Metab. Dispos. 38(3), 415-421 (2010).

29. Englert NA, Luo G, Goldstein JA, Surapureddi S. Epigenetic modification of histone 3 lysine 27: mediator subunit MED25 is required for the dissociation of polycomb repressive complex 2 from the promoter of cytochrome P450 2C9. J. Biol. Chem. 290(4), 2264-2278 (2015).

30. Rieger JK, Reutter S, Hofmann U, Schwab M, Zanger UM. Inflammation-associated microRNA-130b down-regulates cytochrome P450 activities and directly targets CYP2C9. Drug Metab. Dispos. 43(6), 884-888 (2015).

31. Yu D, Green B, Marrone A et al. Suppression of CYP2C9 by microRNA hsa-miR-128-3p in human liver cells and association with hepatocellular carcinoma. Sci. Rep. 5, 8534 (2015).

32. Liu T, Zhong S, Rao F, Xue Y, Qi Z, Wu S. Catheter ablation restores decreased plasma miR-409-3p and miR-432 in atrial fibrillation patients. Europace 18(1), 92-99 (2016).

33. Galenko $\mathrm{O}$, Jacobs $\mathrm{V}$, Knight $\mathrm{S}$ et al. The role of microRNAs in the development, regulation, and treatment of atrial fibrillation. $J$. Interv. Card. Electrophysiol. 55(3), 297-305 (2019).

34. McManus DD, Tanriverdi $\mathrm{K}$, Lin $\mathrm{H}$ et al. Plasma microRNAs are associated with atrial fibrillation and change after catheter ablation (the miRhythm study). Heart Rhythm 12(1), 3-10 (2015).

35. Sardu C, Santamaria M, Paolisso G, Marfella R. microRNA expression changes after atrial fibrillation catheter ablation. Pharmacogenomics 16(16), 1863-1877 (2015).

36. Wang M, Sun L, Ding W, Cai S, Zhao Q. Ablation alleviates atrial fibrillation by regulating the signaling pathways of endothelial nitric oxide synthase/nitric oxide via miR-155-5p and miR-24-3p. J. Cell. Biochem. 120(3), 4451-4462 (2019).

37. Zhou Q, Maleck C, von Ungern-Sternberg SNI et al. Circulating microRNA-21 correlates with left atrial low-voltage areas and is associated with procedure outcome in patients undergoing atrial fibrillation ablation. Circ. Arrhythm Electrophysiol. 11(6), e006242 (2018).

38. Xiang Q, Zhang HX, Wang Z et al. The predictive value of circulating microRNAs for venous thromboembolism diagnosis: a systematic review and diagnostic meta-analysis. Thromb. Res. 181, 127-134 (2019).

39. Wang X, Sundquist K, Svensson PJ et al. Association of recurrent venous thromboembolism and circulating microRNAs. Clin. Epigenetics 11(1), 28 (2019). 
